common to both vaccines were analyzed using a multiplex bead array assay (Luminex). Positive antibody response was defined as at least 2fold increase in prevaccination serotype-specific antibody concentration. Number of serotypes with positive antibody response after prime-booster vaccination were compared between treatment groups and controls using Mann-Whitney $U$ test. In addition, the difference in number of serotypes with positive antibody response between prime and prime-booster vaccination in each treatment group was compared using Wilcoxon signed-ranks test.

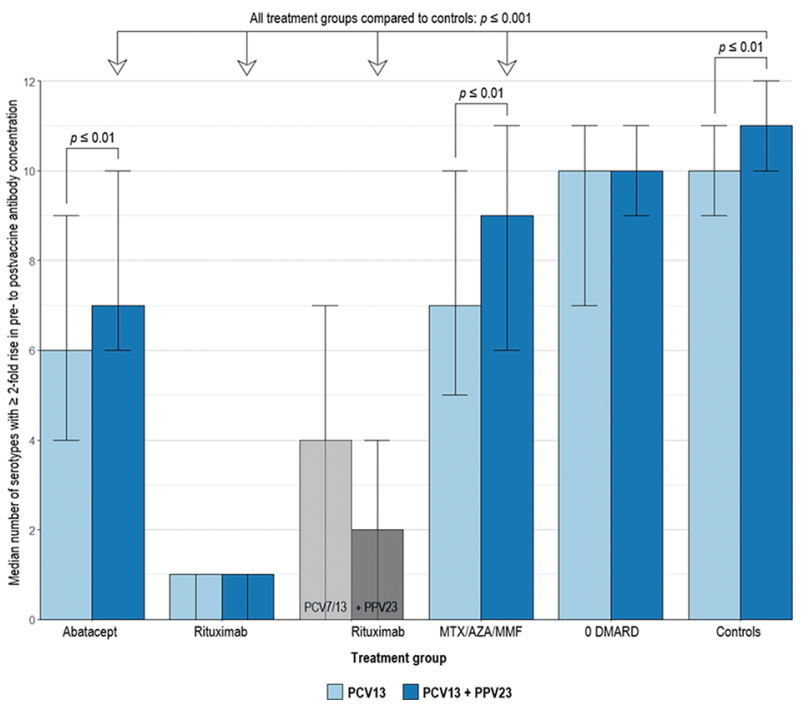

Abstract THU0149 - Figure 1

Results: Compared to PCV alone, the combined schedule with $\mathrm{PCV}+$ PPV23 resulted in increased antibody response for patients treated with abatacept, MTX/AZA/MMF, and controls (all $p \leq 0.01$ ). In patients with rituximab and previous PCV7 or PCV13, booster vaccination with PPV23 did not result in increased antibody levels (Figure: bars marked grey). The numbers of serotypes with a positive antibody response to the combined vaccination with PCV + PPV23 was reduced in patients with rituximab (median 1, $p \leq 0.001$ ), abatacept (median 7, $p \leq 0.001$ ) and MTX/AZA/MMF (median 9, $p \leq 0.001$ ), but not in patients without DMARDs (median 10, $p=0.12$ ), compared to controls (median 11).

Conclusion: Prime-booster vaccination strategy using a combination of a dose of pneumococcal conjugate vaccine followed by a dose of polysaccharide vaccine improved antibody responses in patients with inflammatory rheumatic disease treated with abatacept and traditional DMARDs compared to PCV alone. The combined vaccination resulted in a markedly lower response in rituximab treated patients, and was to a lesser degree reduced in patients with abatacept or traditional DMARDs compared to healthy controls.

Disclosure of Interests: Per Nived: None declared, Göran Jönsson: None declared, Bo Settergren: None declared, Jon Einarsson: None declared, Tor Olofsson: None declared, Johan K Wallman Consultant for: Consultant for AbbVie, Celgene, Eli Lilly, Novartis, and UCB Pharma., Charlotte Sværke Jørgensen: None declared, Meliha C Kapetanovic: None declared DOI: 10.1136/annrheumdis-2019-eular.1125

\section{THU0150 FINGER JOINT CARTILAGE EVALUATED BY ULTRASOUND IN PATIENTS WITH RHEUMATOID ARTHRITIS AND HEALTHY SUBJECTS}

Takehisa Ogura, Ayako Hirata, Norihide Hayashi, Hideki Ito, Sayaka Takenaka, Yuki Inoue, Takaharu Kagtagiri, Chihiro Imaizumi, Yuto Takakura, Hideto Kameda. Toho University, Division of Rheumatology, Department of Internal medicine, Tokyo, Japan

Background: Joint destruction in rheumatoid arthritis (RA) includes both bone and cartilage lesions. Since joint space narrowing (JSN) is not a direct evaluation of cartilage using $\mathrm{X}$-ray, we aimed to examine the validity of ultrasound (US) cartilage evaluation in patients with RA. Objectives:

Methods: The cartilage thickness of bilateral metacarpophalangeal (MCP) and proximal interphalangeal (PIP) joints of the 2 nd to 5 th fingers were visualized from a dorsal view with joints in approximately 90 degrees flexion and measured at the middle portion. Furthermore, one US examiner performed the semiquantitative scoring of the recorded cartilage images in a blinded manner on a scale of $0-2$. In addition, the JSN of the corresponding joints was scored using the van der Heijde-modified Sharp method with a hand X-ray. Continuous variables were analysed using the Mann-Whitney $U$ test. The relationships among the total measurement of cartilage thickness, its semi-quantitative score, and the JSN score were assessed using Spearman's rank correlation coefficients.

Results: One hundred and three patients with RA and 42 healthy subjects were enrolled in this study. The total cartilage thickness was significantly thinner in patients with RA compared to healthy subjects for both the MCP and PIP joints (both $\mathrm{p}<0.001$ ). The semi-quantitative sum of 16 joints ranged from 2 to 26 (median 8) in patients with RA, which was significantly greater than the 0 to 11 (median 4 ) seen in healthy subjects $(p<0.001)$. In patients with $R A$, the semi-quantitative score showed a sig nificant negative correlation with cartilage thickness (rho $=-0.64 ; p<0.001$ ) as well as a significant positive correlation with JSN score (rho $=0.66$; $\mathrm{p}<0.001$ ). In addition, in healthy subjects, semi-quantitative score, but not cartilage thickness was significantly correlated with age (rho $=0.49$; $\mathrm{p}=0.001$ and $\mathrm{rho}=-0.25 ; \mathrm{p}=0.118$, respectively). On the other hand, in RA, these scores showed a significant correlation with RA disease duration but not correlated with age.

Conclusion: A simplified and direct evaluation of finger joint cartilage damage by semi-quantitative US score is valid and useful for patients with RA.

Disclosure of Interests: Takehisa Ogura: None declared, Ayako Hirata: None declared, Norihide Hayashi: None declared, Hideki Ito: None declared, Sayaka Takenaka: None declared, Yuki Inoue: None declared, Takaharu Kagtagiri: None declared, Chihiro Imaizumi: None declared, Yuto Takakura: None declared, Hideto Kameda Grant/research support from: AbbVie, Asahi Kasei Pharma, Astellas, Chugai, Eisai, GlaxoSmith Klein, Mitsubishi-Tanabe, Novartis, Consultant for: AbbVie, Eli Lilly, Novartis, Speakers bureau: AbbVie, Asahi Kasei Pharma, Bristol-Myers, Chugai, Eli Lilly, Janssen, Mitsubishi-Tanabe, Novartis, Pfize

DOI: 10.1136/annrheumdis-2019-eular.6991

\section{THU0151 COMPARISON BETWEEN THREE PROPOSED DEFINTIONS OF DIFFICULT-TO-TREAT/REFRACTORY RHEUMATOID ARTHRITIS IN A COHORT OF BDMARD- TREATED PATIENTS}

Francesca Ometto, Lara Friso, Davide Astorri, Costantino Botsios, Bernd Raffeiner Andrea Doria. University of Padova, Department of Medicine, DIMED, Padova, Italy

Background: A definition of difficult-to-treat/refractory rheumatoid arthritis (RA) (RRA) has not been established yet, nevertheless, RRA is commonly associated with the resistance to multiple bDMARDs $[1,2,3]$.

Objectives: The objective was to evaluate the rate of RRA according to three definitions, the agreement between the definitions and major deter minants of such definitions in a large monocentric cohort of RA patients. Methods: We included RA patients treated with any bDMARD ( $>=1$ year), who started bDMARDs after 2001. Considered definitions of RRA were: B-RAA, according to Buch [3], failure of $>=1$ anti-cytokine (TNF and/or IL6 innibitor) and $>=1$ cell-targeted (B-cell and/or T-cell innibitor) bDMARD; KF-RAA, according to Kearsley-Fleet [2], exposed to $>=3$ bDMARDs classes; DH-RRA, according to de Hair [1], signs and/o symptoms suggestive of inflammatory RA activity (in the study we assumed DAS28 $>=3.2$ or extra-articular manifestations) and failure of $>=1$ csDMARD and $>=2$ bDMARDs. Agreement was measured with Cohen's kappa. To assess variables independently associated with RRA, multivariate regression analysis was used including variables achieving $p<0.10$ in univariate analysis.

Results: Patients included in the study were 572. B-RRA was observed in $165(28.8 \%)$, KF-RRA in $96(16.8 \%)$ and DH-RRA in $57(10.0 \%)$. DHRRA was the most stringent definition. Agreement between the definition was fair: DH-RRA and B-RRA kappa $=0.330, p<0.001$; DH-RRA and KFRRA kappa=0.260, $p<0.001$; B-RRA and KF-RRA kappa=0.275, $p<0.001$. Most common last bDMARD choice in RRA patients differed according to the definition: B-RRA mostly adalimumab (30.9\%) $(p<0.001)$, KF-RRA tocilizumab $(78.1 \%) \quad(p<0.001)$, DH-RRA tocilizumab $(33.3 \%)$ or abatacept $(31.6 \%) \quad(p<0.001) \quad$ (Figure). Multivariate analysis revealed that, as expected, all definitions were affected by the number of bDMARDs, and DH-RRA also by the disease activity. Mean PDN daily dose was associated with B-RRA; lower BMI with KF-RRA; the start of bDMARD treatment in earlier years with KF-RRA and DH-RRA; and radiographic progression (in the 24 months before the study) with DH-RRA (Table). 
Conclusion: RRA was observed in a $20-30 \%$ of RA patients, slightly higher compared to previous evidence [1,2]. Characteristics of patients fulfilling different RRA definitions are diverse. Particularly, disease severity (disease activity and structural damage) was not associated with RRA if the definition considers only the exposure to bDMARDs. Given the large time span of the study period, RRA patients were more frequently those who started bDMARDs in earlier years.

\section{REFERENCES:}

[1] de Hair MJH, et al. Rheumatology 2018;57:1135-1144

[2]. Kearsley-Fleet L, et al. Ann Rheum Dis 2018;77:1405-1412.

[3] Buch MH. Ann Rheum Dis 2018;77:966-969.

Table. Factors associated with three definitions of difficult-to-treat/refractory rheumatoid arthritis (RRA), multivariate analysis.

\begin{tabular}{lccc}
\hline & & OR $(95 \%$ C.I. $)$ & p value \\
\hline B-RRA & & & \\
\hline No. bDMARDs & & $18.77(11.06 ; 31.85)$ & $p<0.001$ \\
Current bDMARD & & $p<0.001$ \\
Prednisone daily dose & per 5 mg increase & $2.15(1.17 ; 2.15)$ & 0.014 \\
Model Constant & & & $p<0.001$ \\
KF-RRA & & & \\
BMl & per 5 unit increase & $0.61(0.35 ; 1.05)$ & 0.072 \\
No. bDMARDs & & $8.69(5.16 ; 14.64)$ & $p<0.001$ \\
bDMARD treatment start year & per year increase & $0.92(0.84 ; 1.01)$ & 0.087 \\
Current bDMARD & & & $p<0.001$ \\
Model Constant & & & 0.069 \\
DH-RRA & & $3.9(2.67 ; 5.71)$ & $p<0.001$ \\
No. bDMARDs & & $0.91(0.83 ; 0.99)$ & 0.026 \\
bDMARD treatment start year & per year increase & $5.55(3.34 ; 9.23)$ & $p<0.001$ \\
DAS28* & per 0.6 unit increase & $2.7(1.21 ; 6.03)$ & 0.015 \\
Radiographic progression ${ }^{* *}$ & & & 0.04 \\
Model Constant & & & \\
& & &
\end{tabular}

OR odds ratio, C.I. confidence interval, *DAS28, mean over the last 12 months;

${ }^{*} \mathrm{mTSS}>=0,5$ over the last 24 months.

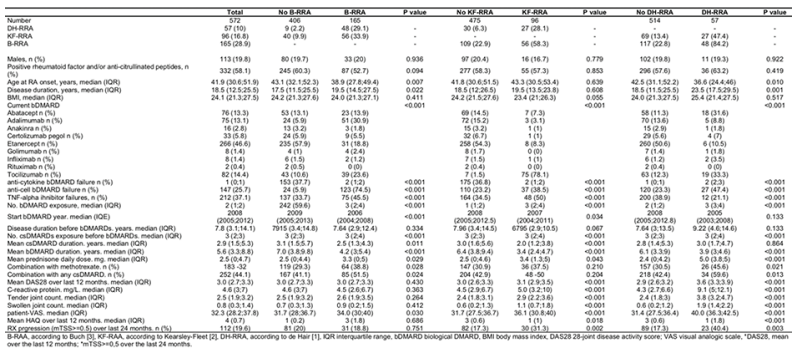

Abstract THU0151 - Figure 1. Characteristics of the patients according to 3 definitions of difficult-to-treat/refractory rheumatoid arthritis (RRA),

Disclosure of Interests: None declared

DOI: 10.1136/annrheumdis-2019-eular.8010

\section{THU0152 SARCOPENIA IS ASSOCIATED WITH PERSISTENT DISEASE ACTIVITY DURING FOLLOW-UP OF RHEUMATOID ARTHRITIS}

Dong-Jin Park, Ji-Hyoun Kang, Haimuzi Xu, Kyung-Eun Lee, Shin-Seok Lee. Cheonnam National University Hospital, Gwangju, Korea, Rep. of (South Korea)

Objectives: This study investigated the prevalence and impact of sarcopenia on clinical outcomes during follow-up in patients with rheumatoid arthritis (RA).

Methods: We enrolled 294 RA patients from a single tertiary center. Data were collected at the time of enrollment and annually thereafter for 3 consecutive years. Sarcopenia was assessed by bioelectrical impedance analysis and defined as a relative skeletal mass index (RSMI) $<5.14 \mathrm{~kg} /$ $\mathrm{m}^{2}$ in women and $<7.40 \mathrm{~kg} / \mathrm{m}^{2}$ in men. Univariate and multivariate analyses were performed to identify the association between sarcopenia and clinical outcome.

Results: Of the 294 RA patients, $8.2 \%$ had sarcopenia at the time of enrollment. Patients with sarcopenia were more likely to be older $(P=0.001)$, male $(P=0.001)$, and smokers $(P=0.001)$, and to have a higher body fat mass $(P=0.034)$ and body fat percentage $(P=0.039)$, and a lower basal metabolic rate $(P=0.013)$, than non-sarcopenic patients. Biologic agents were more commonly prescribed to sarcopenic patients than to non-sarcopenic patients $(P=0.048)$. The sarcopenic patients had higher Physician Global Assessment (PGA) $(P=0.015)$, higher mean and delta tender joints $(P=0.007)$, higher mean and delta swollen joints $(P=0.007)$ and higher mean DAS28-ESR $(P=0.014)$ and DAS28-CRP $(P=0.020)$ scores than the non-sarcopenic patients. In the multivariate analysis, sarcopenia was significantly associated with male gender $(\mathrm{OR}=0.112,95 \%$ Cl: $0.041-0.304, \quad P<0.001)$, mean PGA score $(O R=1.680,95 \% \mathrm{Cl}$ : 1.067-2.646, $P=0.025)$, and mean DAS28-ESR score $(\mathrm{OR}=4.477,95 \%$ Cl: $1.661-12.067, P=0.003$ )

Conclusion: Sarcopenia at baseline was an independent predictor of disease activity during follow-up in Korean patients with RA. Our results provide a rationale for lifestyle interventions designed to maintain normal weight in RA patients and thus help control disease activity.

Disclosure of Interests: None declared

DOI: 10.1136/annrheumdis-2019-eular.3412

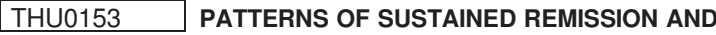 SUBSEQUENT DMARD TAPERING IN EARLY RHEUMATOID ARTHRITIS: DATA FROM THE CANADIAN EARLY ARTHRITIS COHORT}

Maria Powell ${ }^{1}$, Vivian Bykerk ${ }^{2,3}$, Orit Schieir ${ }^{4}$, Janet Pope ${ }^{5}$, Susan J. Bartlett ${ }^{6,7}$, Louis Bessette $^{8}$, Gilles Boire ${ }^{9}$, Carol Hitchon ${ }^{10}$, Edward Keystone ${ }^{11}$, Carter Thorne ${ }^{12}$, Diane Tin ${ }^{12}$, Marie-France Valois ${ }^{6}$, Glen Hazlewood ${ }^{1} .{ }^{1}$ University of Calgary, Calgary, Canada; ${ }^{2}$ Hospital for Special Surgery, New York, United States of America; ${ }^{3}$ Weill Cornell Medical College, New York, United States of America; ${ }^{4}$ University of Toronto Dalla Lana School of Public Health, Toronto, Canada; ${ }^{5}$ Western University, London, Canada; ${ }^{6}$ McGill University, Montréal, Canada; ${ }^{7}$ Johns Hopkins Medicine, Baltimore, United States of America; ${ }^{8}$ Université de Laval, Québec, Canada; ${ }^{9}$ Université De Sherbrooke, Sherbrooke, Canada: ${ }^{10}$ The University of Manitoba, Winnipeg, Canada: ${ }^{11}$ University of Toronto, Toronto, Canada; ${ }^{12}$ Southlake Regional Health Center, Newmarket, Canada

Background: Rheumatoid arthritis (RA) treatment emphasizes aggressive titration of disease-modifying antirheumatic drugs (DMARDs) with the goal of achieving disease remission. This often includes the use of multiple DMARDs in combination, which can have a significant impact on patients lives and add costs to the healthcare system.

Objectives: To describe the patterns of sustained remission and subsequent treatment reduction in usual clinical practice for patients with early RA.

Methods: Patients (age >18) enrolled in the Canadian early ArThritis CoHort (CATCH) between January 2007 to March 2017 were analyzed. $\mathrm{CATCH}$ is a prospective, observational study of patients with early inflammatory arthritis (symptoms $<1$ year) treated in rheumatology clinics across Canada. The analysis cohort included patients with a diagnosis of RA according to the 1987 or 2010 ACR/EULAR classification criteria, active disease at enrolment (DAS28>2.6) and those treated with at least one DMARD or biologic agent within the first three months of study enrolment. We defined sustained remission as achieving a DAS28 $<2.6$ at two consecutive follow-up visits at least six months apart. Reduction of therapy was defined as a minimum of a $25 \%$ dose reduction of conventional synthetic, targeted or biologic DMARDs. Descriptive statistics were used to summarize the time to remission and reductions in DMARD therapy.

Results: Eight hundred and thirty-seven (40\%) of the 2,097 eligible patients achieved sustained remission during the study period. Of these $60 \%$ did so within the first 18 months and $92 \%$ within the first four years (Figure 1). The mean (SD) baseline DAS28 was 5.1 (1.3), and HAQ-DI was $1.0(0.7)$. At the time of remission, $80 \%$ were prescribed methotrexate (55\% subcutaneously), $71 \%$ were prescribed combination therapy with other conventional synthetic DMARDs, and $13 \%$ were prescribed a biologic agent. In the year after attaining sustained remission, $327(39 \%)$ patients reduced treatment in the following pattern (patients may have had more than one change): 250 patients $(30 \%)$ reduced or stopped methotrexate, 196 patients (23\%) reduced or stopped non-methotrexate DMARDs, and 34 patients $(4 \%)$ reduced or stopped biologic agents. Of those that reduced or stopped a biologic, only one was due to side effects. For the 250 patients who reduced or stopped methotrexate, 25 were for a side effect.

Conclusion: Achieving sustained remission occurred in $40 \%$ of early RA patients in usual clinical practice. Treatment reductions following sustained remission occurred in over a third of patients over the next 12 months, and consisted mainly of adjustment in non-biologic DMARDs. 